\title{
Lactobacillus plantarum surface-displayed ASFV (p54) with porcine IL-21 generally stimulates protective immune responses in mice
}

\author{
Xiao-Lei Chen ${ }^{\dagger}$, Jun-Hong Wang ${ }^{\dagger}$ (i), Wei Zhao, Chun-Wei Shi, Kai-Dian Yang, Tian-Ming Niu, Gui-Lian Yang, \\ Xin Cao, Yan-Long Jiang, Jian-Zhong Wang, Hai-Bin Huang, Yan Zeng, Nan Wang, Wen-Tao Yang* and \\ Chun-Feng Wang*
}

\begin{abstract}
African classical swine fever virus (ASFV) has spread seriously around the world and has dealt with a heavy blow to the pig breeding industry due to the lack of vaccines. In this study, we produced recombinant Lactobacillus plantarum (L. plantarum) expressing an ASFV p54 and porcine IL-21 (plL-21) fusion protein and evaluated the immune effect of NC8-pSIP409-pgsA'-p54-pIL-21 in a mouse model. First, we verified that the ASFV p54 protein and p54-plL-21 fusion protein were anchored on the surface of L. plantarum NC8 by flow cytometry, immunofluorescence and Western blotting. Then, the results were verified by flow cytometry, ELISA and MTT assays. Mouse-specific humoral immunity and mucosal and T cell-mediated immune responses were induced by recombinant $L$. plantarum. The results of feeding mice recombinant $L$. plantarum showed that the levels of serum $\lg G$ and mucosal secreted $\lg A(S \lg A)$, the number of CD4 and CD8 T cells, and the expression of IFN- $\gamma$ in CD4 and CD8 T cells increased significantly, and lymphocyte proliferation occurred under stimulation with the ASFV p54 protein. Our data lay a foundation for the development of oral vaccines against ASFV in the future.
\end{abstract}

Keywords: ASFV, p54, Recombinant, L. plantarum, Immune evaluation

\section{Introduction}

African swine fever (ASF) is a highly infectious disease that has caused great economic losses to the animal husbandry economy. Since it was first discovered in Kenya in 1921, 68 countries have been affected by ASF. ASF entered China on August 3, 2018, causing great economic losses to China's animal husbandry (Wang et al. 2018a, b; Zhao et al. 2019). ASFV is the only member of the genus Asfivirus in the Asfarviridae family and spreads between

\footnotetext{
*Correspondence: wangchunfeng@jlau.edu.cn; yangwentao@jlau.edu.cn College of Veterinary Medicine, College of Animal Science

and Technology, Jilin Provincial Engineering Research Center of Animal Probiotics, Key Laboratory of Animal Production and Product Quality Safety of the Ministry of Education, Jilin Agricultural University, 2888

Xincheng Street, Changchun 130118, China

${ }^{\dagger}$ Xiao-lei Chen and Jun-hong Wang Contributed Equally
}

Ornithodoros moubata ticks and warthogs (Bonnet et al. 2020). ASFV infects wild and domestic pigs of all breeds and ages. The clinical manifestations of diseased pigs are fever, skin cyanosis, and obvious bleeding in lymph nodes, kidney, and gastrointestinal mucosa, and the mortality rate is as high as $100 \%$ (Muangkram et al. 2015).

The ASFV genome encodes 150 to 200 proteins, approximately 50 of which are structural proteins. P54 plays an important role in attachment, entry and replication (Jia et al. 2017). Some studies have shown that the p54 protein located in the inner envelope of the virus has good immunogenicity (Petrovan et al. 2020). Some researchers have used the $\mathrm{p} 54$ protein and $\mathrm{p} 30$ protein to construct recombinant subunit vaccines to protect some immunized pigs from the lethal challenge of ASFV (Argilaguet et al. 2012). Recent studies have shown that 
the construction of recombinant Saccharomyces cerevisiae (S. cerevisiae) expressing the $\mathrm{p} 54$ protein can induce mucosal immunity and produce strong antibodies in pigs (Chen et al. 2020). However, due to the complexity of the ASFV virus, the existence of multiple genotypes and the limited effect on protective immunity, no ASFV vaccine is available (Rock. 2017; Sang et al. 2020). The findings of researchers in 2019 provide a comprehensive molecular model for the capsid structure of ASFV. It is hoped that this information will contribute to the development of future strategies, including vaccines (Liu et al. 2019).

Interleukin 21 (IL21) is a cytokine mainly produced by differentiated mature $\mathrm{CD} 4^{+} \mathrm{T}$ cells and natural killer $\mathrm{T}$ cells (Battaglia et al. 2014; Wang et al. 2015). Some studies have found that IL-21 can enhance the expression of CD86 in mouse B cells so that it can provide superior $\mathrm{T}$ cell costimulatory ability (Attridge et al. 2014; Tangye and Ma 2020). There is evidence that IL21 participates in B cell activation and proliferation through its specific receptor (IL21R) and regulates germinal center and humoral immunity (Tangye et al. 2017), while lack of IL-21 leads to memory B cell deficiency (Erman et al. 2015; Moens and Tangye 2014). In summary, IL-21 is a potential vaccine adjuvant.

Lactic acid bacteria are the most commonly used probiotics, which can maintain intestinal homeostasis and enhance immunity (Vinusha et al. 2018). Compared with traditional attenuated vaccines, recombinant $L$. plantarum based on probiotics is considered to be an excellent tool for veterinary vaccines (Yang et al. 2017), which can induce a strong immune response by surface anchored expression of antigen, while L. plantarum NC8 isolated from silage has a high ability to express foreign protein (Yang et al. 2017). To construct recombinant $L$. plantarum that can express foreign antigens, our laboratory used the truncated anchor sequence of poly- $\gamma$ glutamate synthase A' (PGSA') (Cai et al. 2017), which is a transmembrane protein from Bacillus subtilis and has high display efficiency (Lei et al. 2015). For instance (Jiang et al. 2017), L. plantarum has been used to express conserved M2E and HA2 fusion antigens to induce protection against influenza viruses (Yang et al. 2018a, b, c). Expression of the S-DCpep fusion protein against transmissible gastroenteritis virus (Yang et al. 2018a, b, c) expressing the porcine epidemic diarrhea virus $\mathrm{S}$ gene can improve the immune response of the body (Jin et al. 2018). In this study, a recombinant $L$. plantarum strain that can express the ASFV p54 protein and p54-pIL-21 fusion protein was designed to evaluate the immune effect of mice in order to lay a theoretical foundation for the development of oral vaccines.

\section{Materials and methods}

Animals and ethical statement

The animals used in this experiment were purchased from HFK Bioscience Co., Ltd. (Beijing, China). Pathogen-free female C57BL/6 mice aged 6 to 8 weeks were raised in SPF rooms. The whole animal experiment met the requirements of the Animal Management and Ethics Committee of Jilin Agricultural University.

\section{Construction of recombinant $L$. plantarum}

To construct recombinant $L$. plantarum, the gene sequences of porcine interleukin IL-21 (pIL-21) (gene number: AB073020.1) and p54 (gene number: 22220355) of African classical swine fever virus were synthesized and purified by GENEWIZ (Jiangsu, China) and carried out code optimization (Additional file 1). Then, we digested these constructs with XbaI (Takara, Japan) and HindIII (Takara, Japan), cloned the p54 gene and p54-pIL-21 fusion gene into the pSIP-409-pgsA' vector, and transformed the positive plasmid into L. plantarum strain NC8 (CCUG 61730). Two recombinant $L$. plantarum strains, NC8-pSIP409-pgsA'-p54 and NC8pSIP409-pgsA'-p54-pIL-21, were obtained. They were sequenced and identified by Shanghai Shenggong Biotechnology Co., Ltd. (Shanghai, China).

\section{Preparation of anti-p54 antibody}

The p54 gene sequence was ligated into the pET28a expression vector and transformed into BL21 to obtain recombinant Escherichia coli BL21-pET-28a-p54. p54 protein expression was induced by IPTG (100 mM) (Sigma, Japan). The p54 protein was purified and recovered. Rabbit polyclonal antibodies were obtained by immunizing rabbits with purified p54 protein and used to detect the expression of bacterial target genes.

\section{Western blotting}

To detect the expression of p54 and p54-pIL-21 antigens in L. plantarum NC8, NC8-pSIP409-pgsA'-p54 and NC8pSIP409-pgsA'-p54-pIL-21 were cultured with $10 \mu \mathrm{g} / \mathrm{mL}$ erythromycin and $50 \mathrm{ng} / \mathrm{mL}$ sakacin P inducer (SppIP) at $30{ }^{\circ} \mathrm{C}$ for $9 \mathrm{~h}$. After separation by SDS-PAGE (10\% acrylamide), the bacterial protein was transferred to a nitrocellulose membrane and incubated with the polyclonal rabbit anti-p54 antibody and then a goat anti-rabbit secondary antibody coupled with horseradish peroxidase (HRP) (CST, USA). After washing, protein detection was visualized by enhanced chemiluminescence (ECL, USA) on an Amersham Imager (General Electric Company).

Flow cytometry assay and immunofluorescence detection The recombinant strain $L$. plantarum $(0.5 \mathrm{~mL})$ induced by the above method was resuspended in PBS and diluted 
to the appropriate $\mathrm{OD}$ value, incubated with the rabbit anti-p54 antibody(BD Bioscience, U.S.A) at $4{ }^{\circ} \mathrm{C}$ for $1 \mathrm{~h}$, and then stained with a goat anti-mouse IgG labeled with FITC at $4{ }^{\circ} \mathrm{C}$ for $1.5 \mathrm{~h}$. After washing with PBST, the recombinant bacterial cells were evaluated by flow cytometry (BDLSR Fortessa cells (USA) and fluorescence microscopy (Leica DMI8, Germany)).

\section{Immunization}

To evaluate the immune effect of recombinant $L$. plantarum NC8-pSIP409-pgsA'-p54 and NC8-pSIP409pgsA'-p54-pIL-21, 40 C57BL/6 mice were divided into four groups. The mouse group was immunized with $200 \mu \mathrm{L}$ with colony-forming units $\left(1 \times 10^{9} \mathrm{CFU}\right)$ of NC8-pSIP409-pgsA', NC8-pSIP409-pgsA'-p54 or NC8pSIP409-pgsA'-p54-pIL-21 suspension by oral gavage. The control group was given $0.9 \%$ normal saline $(200 \mu \mathrm{L})$ by the same method. The mice were first immunized on the 1st, 2nd and 3rd days, then received booster immunization on the 14th, 15th and 16th days, and finally boosted again on the 28th, 29th and 30th days. The feces and serum of each mouse were collected for ELISA detection on the 13th, 27th and 43rd days.

\section{Flow cytometry}

Nine mice in each group were euthanized on the 14th day after the last immunization. Single-cell suspensions of spleen (SP), mesenteric lymph nodes (MLNs) and Peyer's patches (PPs) of all groups of mice were prepared as previously described (Huang et al. 2018). After preparing single-cell suspensions from MLNs and PPs, we transferred $10 \mu \mathrm{L}$ of anti-B220 antibody (BD Bioscience, USA) into a test tube containing $1 \times 10^{6}$ cells, mixed the solution well and stained the cells in the dark at $4{ }^{\circ} \mathrm{C}$ for $30 \mathrm{~min}$. We added $1 \mathrm{~mL}$ of phosphate-buffered saline (PBS) to the collected samples. The cell suspension was centrifuged at $2000 \mathrm{rpm}$ and $4{ }^{\circ} \mathrm{C}$ for $5 \mathrm{~min}$, and the supernatant was discarded. The above steps were repeated. The cells were then fixed and permeabilized, centrifuged twice, and stained for $30 \mathrm{~min}$ with $10 \mu \mathrm{L}$ of anti-IgA antibody (BD Bioscience, USA) at $4{ }^{\circ} \mathrm{C}$ in the dark using the same procedure described above. Single-cell suspensions of MLNs and SPL were transferred to a 48-well cell culture plate, and the cells were incubated with the p54 protein for $8 \mathrm{~h}$. Then, inhibitors were added for $3 \mathrm{~h}$. The cells were centrifuged twice, and $10 \mu \mathrm{L}$ of anti-CD3, anti-CD4 and anti-CD8 antibodies (BD Biosciences, USA) were added. The cells were then fixed, permeabilized, centrifuged twice, and mixed with $10 \mu \mathrm{L}$ anti-IFN- $\gamma$ antibody (BD Biosciences, USA) for $30 \mathrm{~min}$ at $4{ }^{\circ} \mathrm{C}$ in the dark. BD fluorescence-activated cells were sorted and analyzed by FACS in an LSRFortessa analyzer (BD Bioscience, USA). All data were analyzed using FlowJo 7.6 software.

\section{Enzyme-linked immunosorbent assay}

The presence of antigens that specifically bound to IgG and IgA antibodies in serum and Fecal supernatant was assessed by Enzyme-linked immunosorbent assay (ELISA), with some minor alterations (Shi et al. 2014). Briefly, 96-well polystyrene microtiter plates were coated with $1 \mu \mathrm{g}$ p54 antigen using carbonate-bicarbonate buffer (pH9.6) and incubated overnight at $4{ }^{\circ} \mathrm{C}$. Then sealed with $150 \mu \mathrm{L}$ of blocking solution (PBST containing 10\% bovine serum albumin) at $37^{\circ} \mathrm{C}$ for $1 \mathrm{~h}$. The wells were washed three times with PBST. The diluted samples were added to the well, incubated at $37^{\circ} \mathrm{C}$ for $2 \mathrm{~h}$, and then washed three times with PBST. $100 \mu \mathrm{L}$ of anti-mouseHRP conjugate (CST, USA) was added to all the wells at a dilution of 1:5000 and incubated for $1 \mathrm{~h}$. After washing four times with wash buffer, the plate was developed with $0.02 \% \mathrm{O}$-phenylenediamine and $0.015 \% \mathrm{H}_{2} \mathrm{O}_{2}$ (Zymed) in substrate buffer ( $15 \mathrm{mM}$ citrate buffer $\mathrm{pH} 5.6)$, and the reaction was stopped after 10 min with $2 \mathrm{~N} \mathrm{H} 2 \mathrm{SO} 4$. The absorbance was read at $492 \mathrm{~nm}$. Finally, termination solution is added to terminate the reaction. The OD value was determined by an enzyme labeling instrument. The final titer was evaluated as the highest dilution, resulting in twice the absorbance of the sample background.

\section{Lymphocyte proliferation test}

To evaluate the proliferation of primary immunized lymphocytes, MLN cells $\left(2 \times 10^{5}\right.$ cells $)$ and SLP cells $\left(2 \times 10^{5}\right.$ cells) were placed in 96-well plates, and p54 antigen $(5 \mu \mathrm{g} / \mathrm{mL})$ was added to each well. After $72 \mathrm{~h}$, the cells were removed, and $20 \mu \mathrm{L}$ of MTS was added to each well to stop the reaction. After $4 \mathrm{~h}$, the OD value was detected by an enzyme labeling instrument.

\section{Statistical analysis}

All the data in the experiment came from at least three independent experiments and are expressed as the average \pm SEM. GraphPad Prism 5.0 software was used to test the differences. $\mathrm{P}<0.05$ was considered to represent a significant difference. Analysis of variance (ANOVA) with Tukey's multiple comparison test was used to evaluate the significance.

\section{Results}

\section{Construction of plasmids and expression of target genes in vitro}

Two recombinant L. plantarum NC8-pSIP409-pgsA'-p54 and NC8-pSIP409-pgsA'-p54-pIL-21 strains (Fig. 1A) were successfully constructed. Rabbit polyclonal antibodies were used to bind to the target protein p54 and p54-pIL-21 fusion protein and then incubated with a goat anti-rabbit secondary antibody (HRP labelled) (Fig. 1B). The WB results showed that the target band 

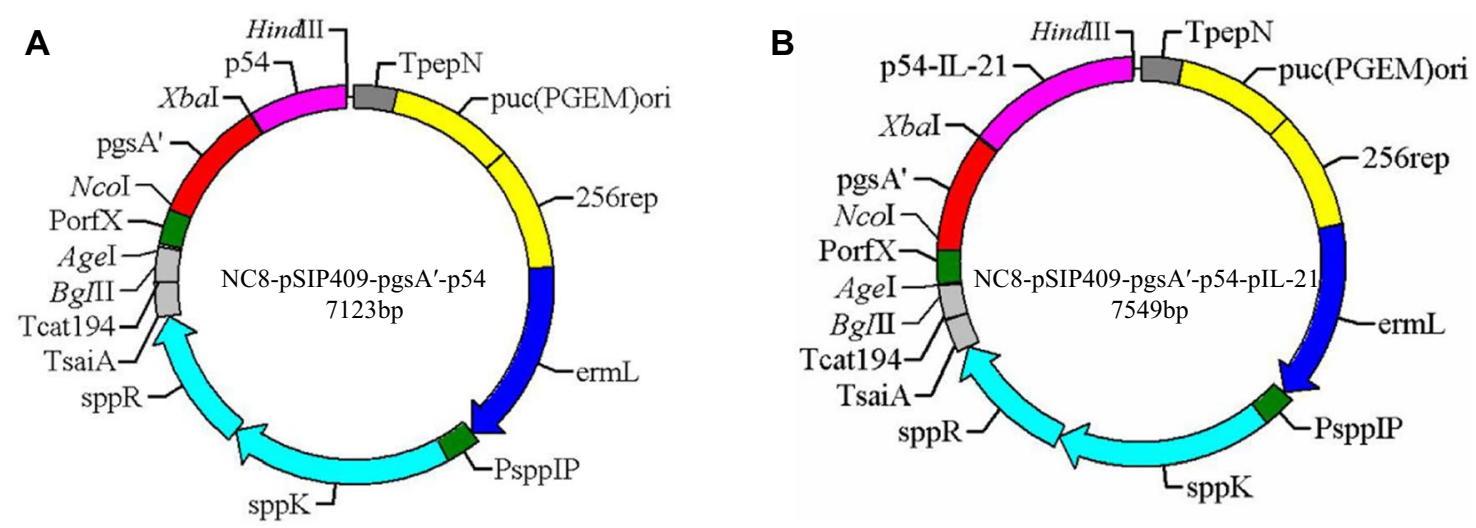

C

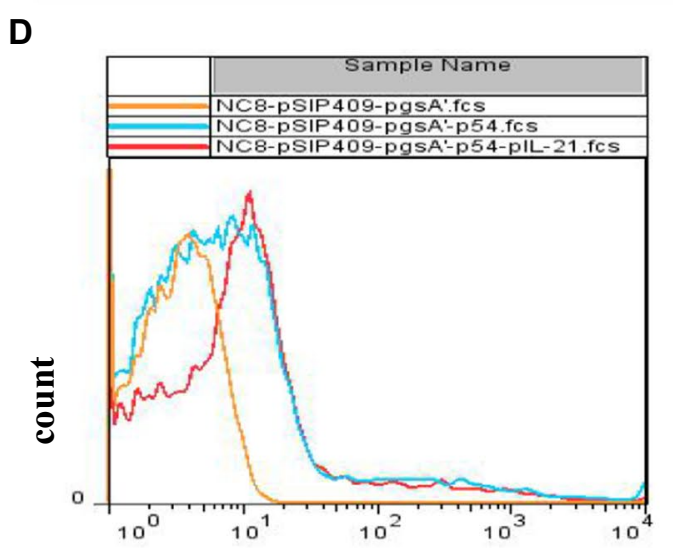

FITC

E NC8-pSIP409-pgsA'

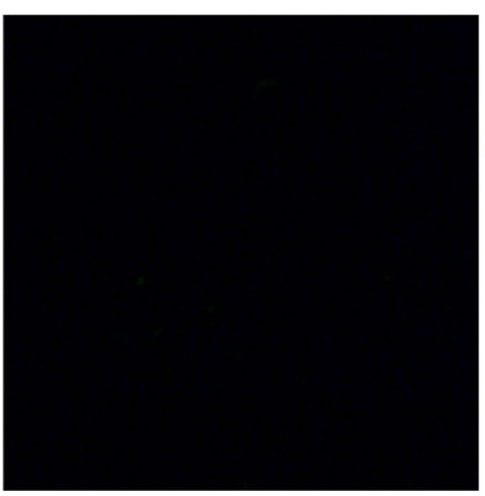

$35 \mathrm{kDa}$

$25 \mathrm{kDa}$
$\mathbf{F}$

$$
\text { NC8-pSIP409-pgsA'-p54 }
$$

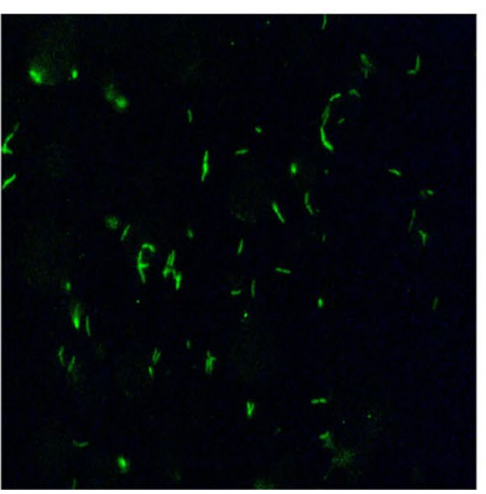

G

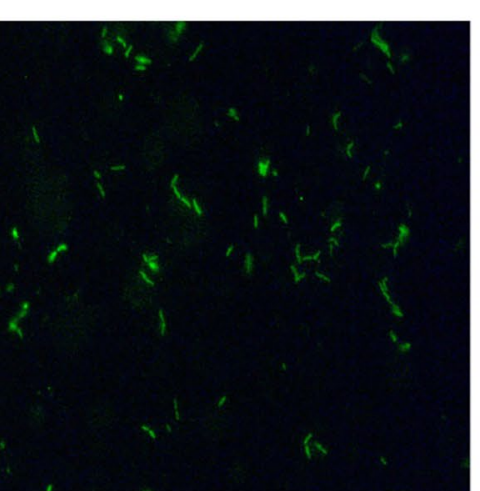

Fig. 1 A A map of the plasmid NC8-pSIP409-pgsA'-p54; B a map of the plasmid NC8-pSIP409-pgsA'-p54-pIL-21; C the expression of the recombinant L. plantarum ASFV p54 protein and p54-pIL-21 fusion protein was verified by Western blot, lane 1: NC8-pSIP409-pgSA', lane 2: NC8-pSIP409-pgsA'-p54, lane 3: NC8-pSIP409-pgsA'-p54-pIL-21; D the expression of recombinant Lactobacillus plantarum was verified by immunofluorescence; $\mathbf{E}$ the anchored expression of the ASFV p54 protein and p54-plL-21 fusion protein on the surface of Lactobacillus plantarum was verified by immunofluorescence

was consistent with the expected size, which proved that the rabbit-specific antibody was obtained by immunizing rabbits with purified p54 antigen and that the recombinant $L$. plantarum successfully expressed foreign protein
(Fig. 1C). The results of immunofluorescence detection showed that the FITC-labeled secondary antibody could combine with the rabbit primary antibody; thus, the surface-anchored expression of the $\mathrm{p} 54$ protein and 
p54-pIL-21 fusion protein of L. plantarum was ideal, and the expression efficiency was high (Fig. 1D). The protein expression of $L$. plantarum was detected by flow cytometry after induction. The results showed the anchored expression (Fig. 1E) of L. plantarum on the surface of the bacteria.

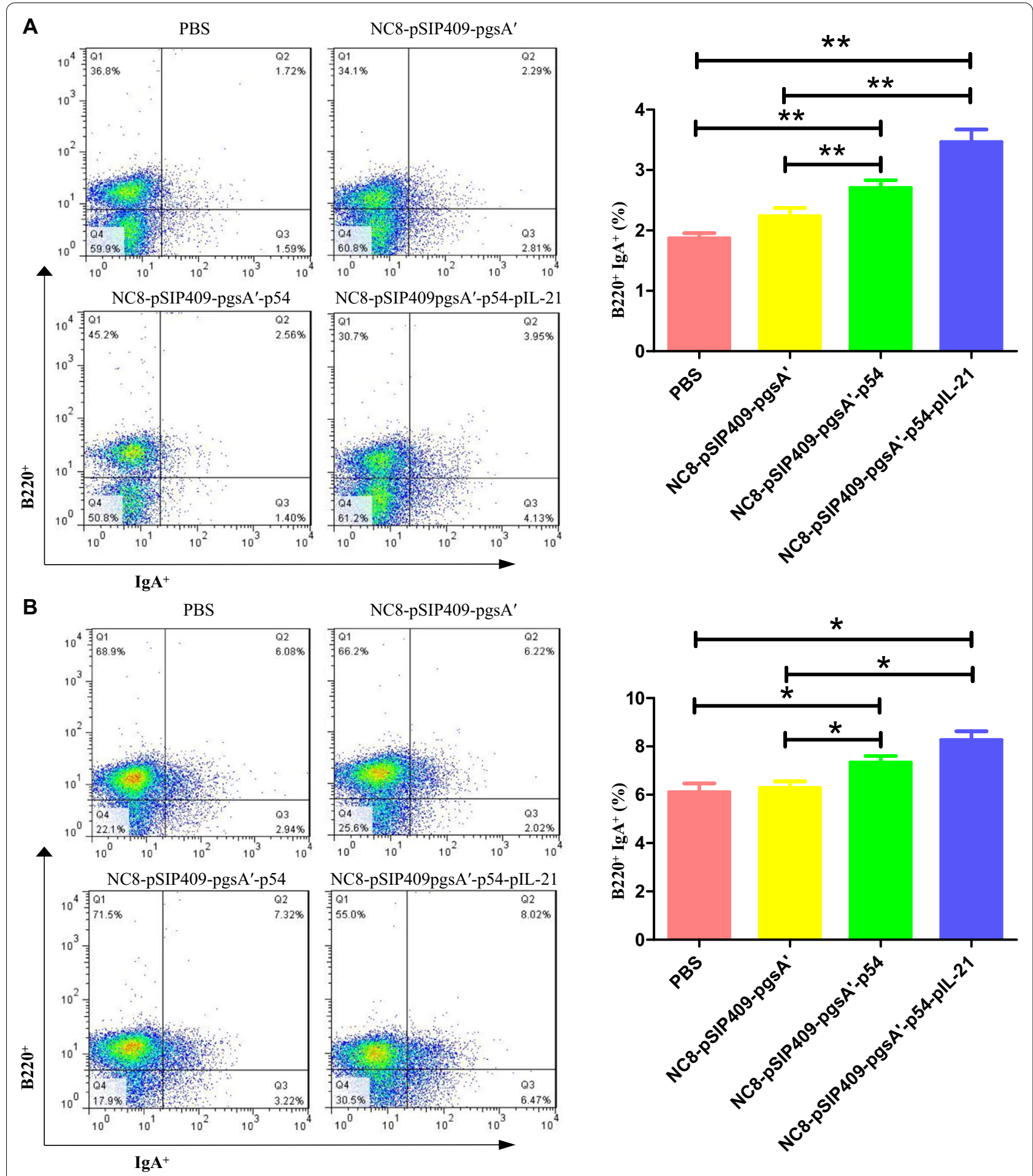

Fig. 2 A Statistical analysis of lymphocyte expression of B220 and lgA in mouse PPs; B statistical analysis of lymphocyte expression of B220 and lgA in mouse MLNs 
Mucosal immunity induced by recombinant $L$. plantarum Recombinant $L$. plantarum significantly enhanced the detection of $\mathrm{B}^{2} 20^{+} \mathrm{IgA}^{+}$cells in PPs (Fig. 2A) and MLNs (Fig. 2B). The results of IgA detection in the feces of mice showed that after the first immunization, the IgA content in the feces of the mice fed NC8-pSIP409-pgsA'-p54pIL-21 was significantly higher than that of the control mice. After the third immunization, the IgA content in the feces of mice fed NC8-pSIP409-p54-IL-21 was significantly higher than that of mice fed PBS (Fig. 3A). The results of IgA detection in the feces of mice showed that there was no significant change in the mice fed PBS after three immunizations; the IgA content in the feces of the mice fed NC8-pSIP409-pgsA' and the mice fed NC8pSIP409-pgsA'-p54 increased slightly, but the difference was not significant; the IgA content in the feces of mice fed NC8-pSIP409-pgsA'-p54-pIL-21 increased significantly (Fig. 3B).

\section{Increase in IgG content in serum induced by recombinant L. plantarum}

The results of serum IgG detection showed that there was no significant difference in serum IgG among the four groups after the first immunization. The IgG content in the serum of mice fed NC8-pSIP409-pgsA'-p54 and NC8-pSIP409-pgsA'-p54-pIL-21 was slightly higher than that of the control and blank mice, but the difference was not significant. After the second immunization, the IgG content in the serum of mice fed NC8-pSIP409p54-pIL-21 was significantly higher than that of the control mice. After the third immunization, the IgG content in the serum of mice fed NC8-pSIP409-pgsA'-p54 was significantly higher than that of mice fed PBS or NC8pSIP409 (Fig. 3C). The results of serum IgG detection showed that there was no significant change in the serum IgG content of mice fed PBS after three immunizations, but the IgG content in the serum of mice fed the NC8pSIP409-pgsA' group increased slightly, but the difference was not significant, while the IgG content in the serum of mice fed NC8-pSIP409-pgsA'-p54 and mice fed NC8-pSIP409-pgsA'-p54-pIL-21 increased significantly (Fig. 3D).

\section{Cellular immune response induced by recombinant $L$. plantarum}

Recombinant $L$. plantarum enhanced immunity, and the contents of $\mathrm{CD}^{+}{ }^{+} \mathrm{CD}_{4}{ }^{+}$and $\mathrm{CD}^{+}{ }^{+} \mathrm{CD}^{+}{ }^{+} \mathrm{T}$ cells in SP lymphocytes increased significantly (Fig. 4A). The MLN is one of the main mucosa-associated lymphoid tissues, in which the level of activated lymphocytes is an important marker for evaluating immunity. IFN- $\gamma$ can be produced when $T$ cells are stimulated by antigens, which can widely neutralize viruses and play an important role in immune regulation. Recombinant L. plantarum enhanced the detection of $\mathrm{CD} 4^{+} \mathrm{IFN}-\gamma^{+}$and $\mathrm{CD}^{+}{ }^{+}$IFN- $\gamma^{+}$T cells in the MLN (Fig. 4B). The results of

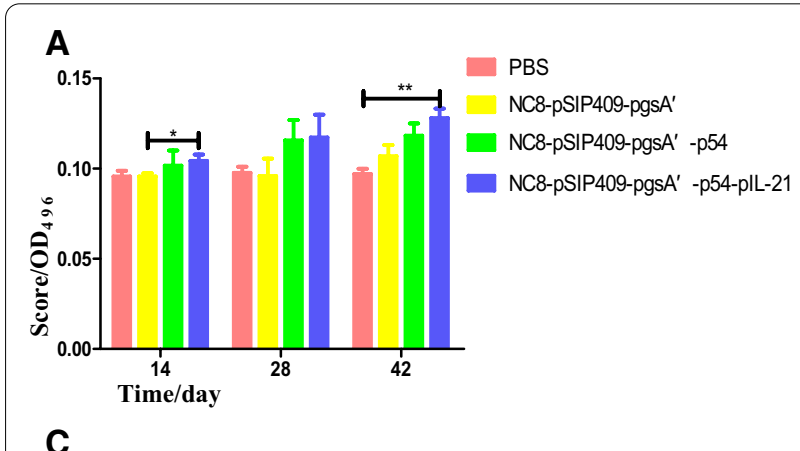

B
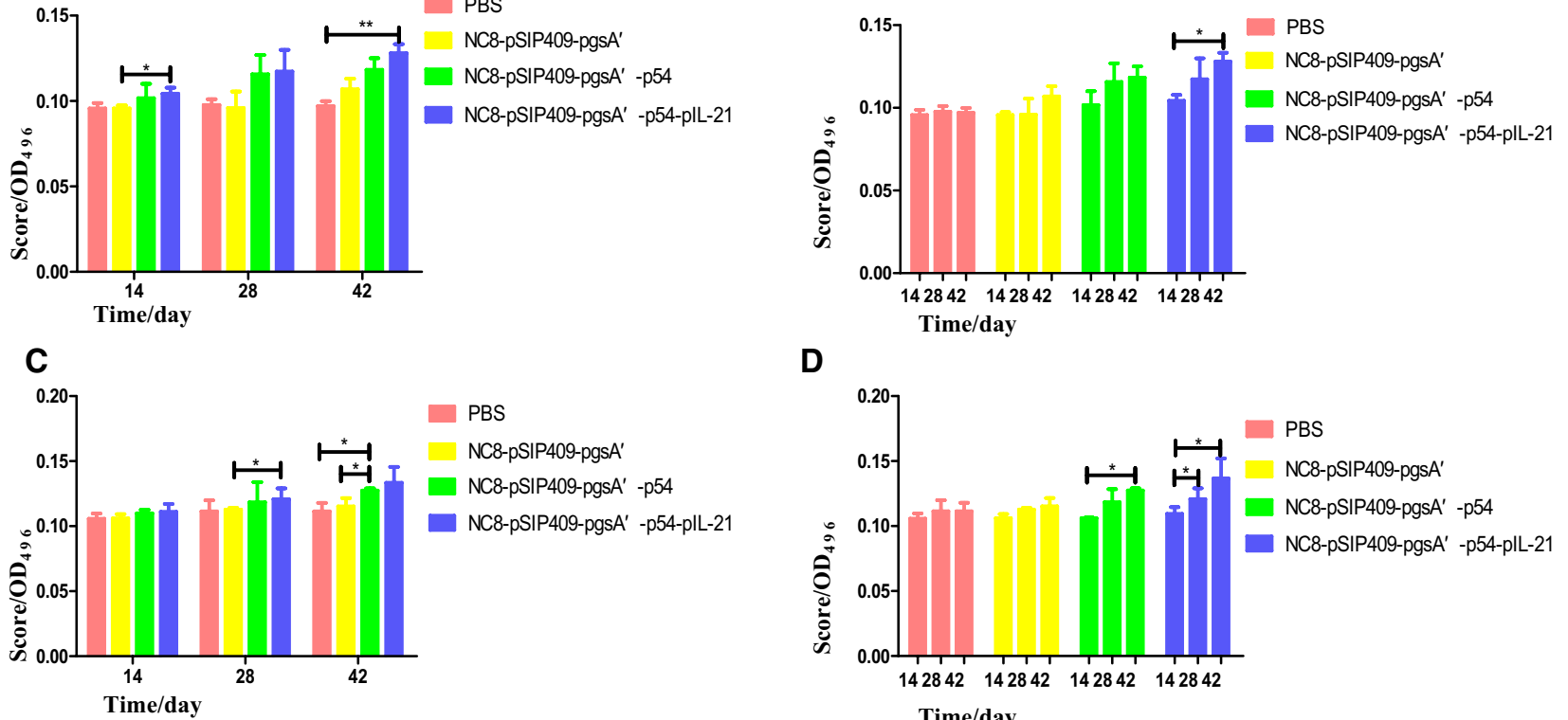

D

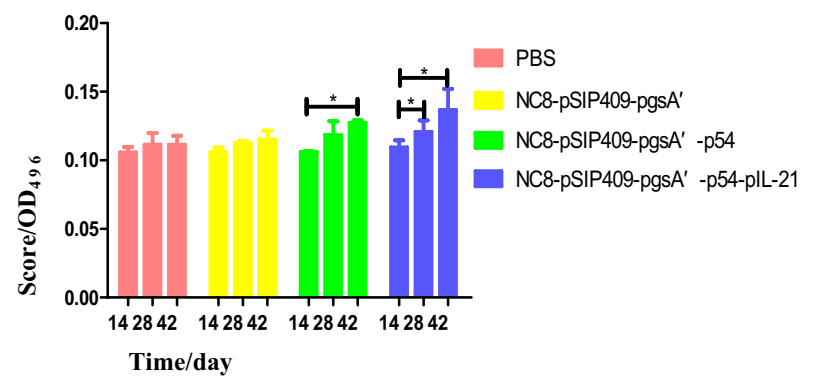

Fig. 3 A Comparison of the increase in fecal IgA content in the four groups of mice after three immunizations. B Comparison of the increase in the fecal IgA content of the four groups of mice after each immunization. C Comparison of the increase in the fecal lgG content of the four groups of mice after three immunizations. D The increase in the fecal lgG content of the four groups of mice after each immunization 

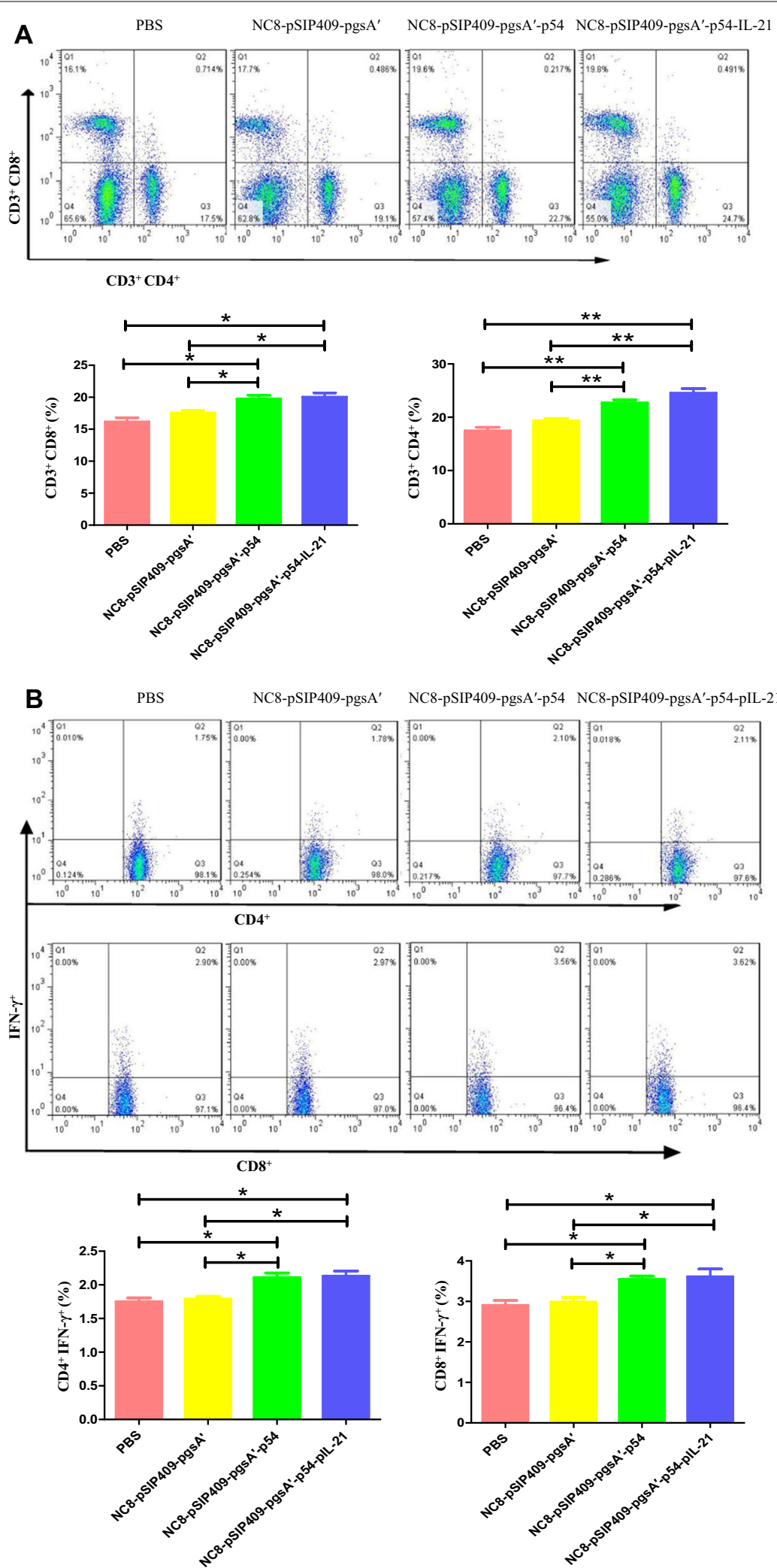

Fig. 4 A Changes in the detection of $\mathrm{CD}^{+} \mathrm{CD}^{+}$and $\mathrm{CD} 3^{+} \mathrm{CD} 4^{+} \mathrm{T}$ cells in the SP; $\mathbf{B}$ changes in $\mathrm{CD} 4^{+} \mathrm{IFN}-\gamma^{+}$and $\mathrm{CD} 8^{+}$IFN- $\gamma^{+} \mathrm{T}$ cells in the MLN 

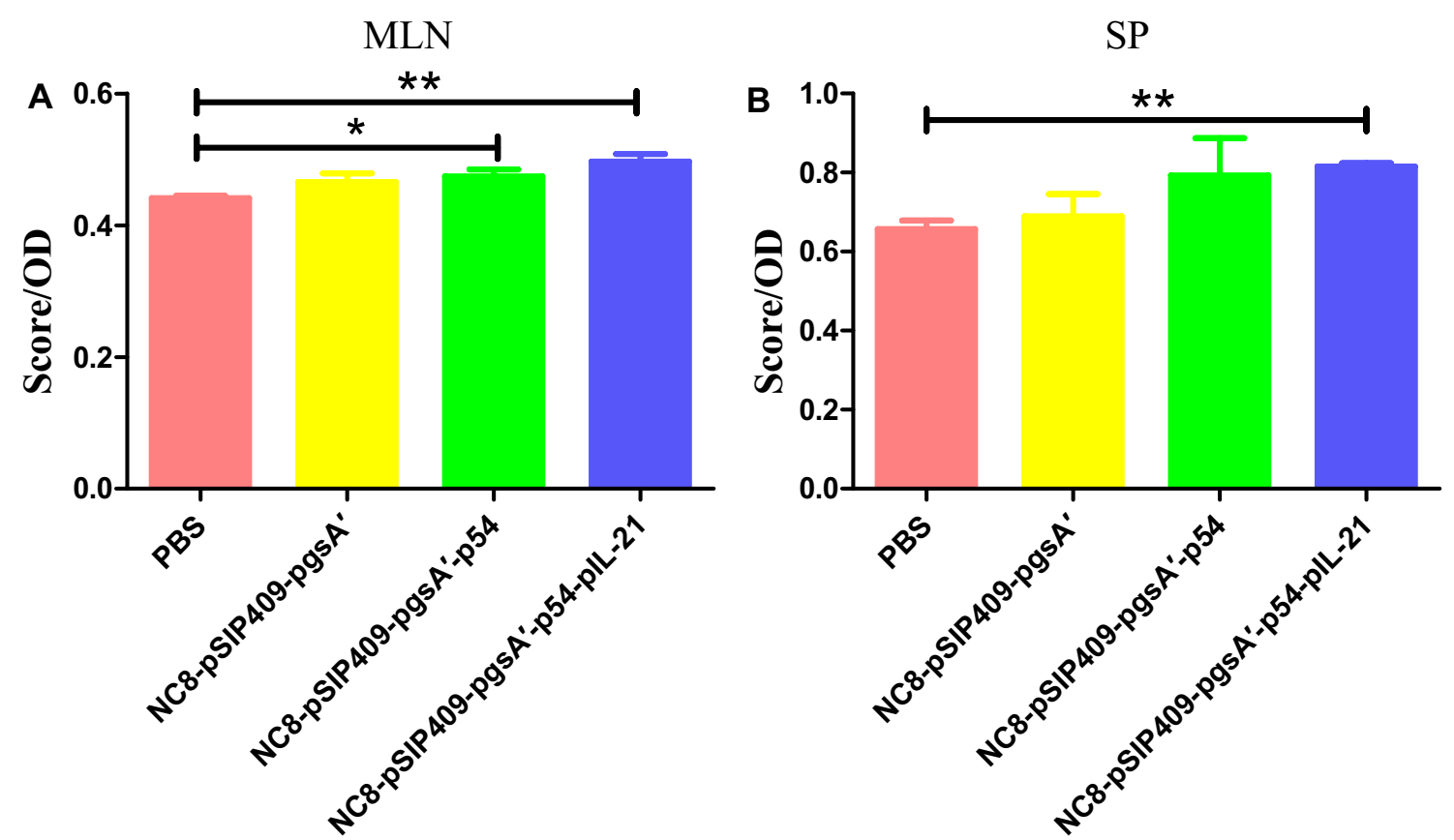

Fig. 5 A The results of lymphocyte proliferation in the MLN. B The results of lymphocyte proliferation in the SP

MLN lymphocyte proliferation showed that the mice fed recombinant $L$. plantarum NC8-pSIP409-pgsA'-p54 and NC8-pSIP409-pgsA'-p54-pIL-21 had significantly greater proliferation than those fed PBS (Fig. 5A). The results of SP lymphocyte proliferation showed that the mice fed recombinant L. plantarum NC8-pSIP409-pgsA'-p54pIL-21 had significantly greater proliferation than those fed PBS (Fig. 5B). There are a certain number of memory lymphocytes in mice stimulated by $\mathrm{p} 54$ antigen and p $54-$ pIL-21 fusion antigen that can proliferate and produce specific antibodies to bind to them when recognizing antigens.

\section{Discussion}

ASF is a complex pig disease that is poorly controlled in most affected areas due to a lack of vaccines. Therefore, it is urgent to develop a reasonably protective ASF vaccine (Arias et al. 2017). It is known that all kinds of ASFV vaccines have not achieved good results, but some scholars have proposed the advantages and feasibility of subunit vaccines (Gaudreault and Richt 2019). At the same time, this experiment used L. plantarum to express the ASFV P54 antigen in mice and achieved a good immune effect.

Vaccination through the mucosal route is helpful to target the induced site of the SIgA response and greatly enhance the mucosal immune response, which is the most effective way to trigger a protective mucosal immune response (Boyaka. 2017; Jin et al. 2019). For example, a human mucosal antiinfluenza vaccine can induce high local humoral and cellular immune responses (Calzas and Chevalier 2019); a mucosal vaccine constructed with the HIV envelope protein induces HIV-specific immunity in the blood and mucosa where the virus enters (Chege et al. 2017). Studies have shown that microbial antigens can induce antigen-specific mucosal and systemic immune responses in mice and can be used to prevent microbial infections in the intestinal tract (Fujimoto et al. 2019). The L. plantarum strain used in this study can protect the intestinal barrier and activate the proliferation of intestinal epithelial cells (Hou et al. 2018). L. plantarum has good application prospects as a mucosal vaccine carrier. In our experimental results, we found that recombinant $L$. plantarum can significantly increase the secretion of SIgA from the intestinal mucosa. In the past decade, the successful development of mucosal vaccines against influenza virus and rotavirus infection has increased people's interest in this field, and people have high hopes for new mucosal vaccines (Lycke 2012).

It is well known that the increase in IgG content in body fluid helps to improve the antiviral activity of the body. Specific IgG was detected from 15 to 21 days after pigs were immunized with African classical swine fever live attenuated vaccine and maintained at a high level during the experiment (Sánchez-Cordón et al. 2018). An adenovirus vector vaccine containing the ASFV antigen 
can induce an increase in IgG content in the body fluid of pigs and has good immunogenicity (Lokhandwala et al. 2019). In our study, the IgG content in the blood of mice in the experimental group increased significantly after each immunization and reached the highest value 14 days after the third immunization, which was consistent with the humoral immunity induced by the subunit vaccine expressing foot-and-mouth disease in Holstein cattle (Sitt et al. 2019). Some researchers immunized mice with the ASFV structural protein $\mathrm{p} 72$ recombinant Newcastle disease virus vaccine to produce a high-titer specific IgG antibody (Chen et al. 2016). Therefore, the increase in IgG content in body fluid contributes to the increase in anti-ASFV activity.

ASFV infection of animal lymphocytes results in a decrease in lymphocyte activity and can negatively regulate the expression of interferon, which plays an important role in viral immune escape (Wang et al. 2018a, b). The proliferation of $\mathrm{CD}^{+}$and $\mathrm{CD} 8^{+} \mathrm{T}$ cells is affected (Childerstone et al. 1998). It is known that helper $\left(\mathrm{CD}_{4}^{+}\right)$ $\mathrm{T}$ cells play an important role in inducing the mucosal antibody response (Aljurayyan et al. 2018). In addition, studies have shown that $\mathrm{CD} 8^{+}$lymphocyte subsets play an important role in ASFV protective immunity (Takamatsu et al. 2013). Protective immunity against African classical swine fever virus is lost when $\mathrm{CD}^{+} \mathrm{T}$ lymphocytes are depleted in the body (Oura et al. 2005). Some studies have shown that interferon- $\gamma$ can inhibit the replication of African classical swine fever virus (Fan et al. 2020), and $T$ cells proliferate and increase the secretion of interferon- $\gamma$ in mice immunized with ASFV structural protein (Chen et al. 2016). Interestingly, in our study, we also found that the number of activated $\mathrm{CD} 4^{+} \mathrm{T}$ cells and $\mathrm{CD}^{+} \mathrm{T}$ cells in the experimental group increased, the secretion of IFN- $\gamma$ in $\mathrm{CD}^{+}$and $\mathrm{CD}^{+}{ }^{+} \mathrm{T}$ cells increased, and lymphocytes proliferated under stimulation with the ASFV p54 antigen. Other studies have shown that vaccines that induce a high-intensity $\mathrm{CD} 8^{+} \mathrm{T}$ cell response may enhance the protective effect against HIV virus (Petitdemange et al. 2019).

Interleukin-21 (IL-21) plays an important role in both innate and acquired immune responses, and its expression is significantly increased in a variety of viral infections. The specific receptor IL-21R is produced and localized on the surface of $\mathrm{T}$ and $\mathrm{B}$ cells and natural killer (NK) cells, which plays a key role in the proliferation and differentiation of these immune effector cells (Shoraka et al. 2019). IL-21 can also increase the number of helper $\left(\mathrm{CD}^{+}\right) \mathrm{T}$ cells and germinal center B cells, thus increasing the immunity of the body (Zhang et al. 2016). Some studies have shown that exogenous methods to increase IL-21 production or increase cellular IL-21 production can limit the degree of initial HIV-1 infection (Ortega et al. 2018). A number of studies have shown that IL-21 plays an important role in antiviral humoral immunity (Rasheed et al. 2013). Because of the high homology between porcine IL-21 and mouse IL-21 (approximately 80\%), the immune effect of mice fed recombinant $L$. plantarum expressing p54 antigen and pIL-21 in our experiment is also the most significant, and related experiments will be carried out in pigs in the future.

In this study, we produced recombinant $L$. plantarum expressing a ASFV p54 and p54-pIL-21 fusion protein and evaluated the immune effect of NC8-pSIP409-pgsA'-p54 and NC8-pSIP409-pgsA'-p54-pIL-21 in a mouse model. The results showed that recombinant $L$. plantarum could improve the humoral immunity, cellular immunity and mucosal immunity of mice, which laid a theoretical foundation for the development of oral vaccines against ASFV. For the phenomenon of ASFV infection through the mucosa, the mucosal vaccine may have a certain effect on blocking the virus and provide guidance for our further experiments in pigs.

\section{Supplementary Information}

The online version contains supplementary material available at https://doi. org/10.1186/s13568-021-01275-9.

Additional file 1. The optimized sequence of p54.

\section{Acknowledgements}

We thank Chun-Feng Wang and Wen-Tao Yang for their support in the experiments.

\section{Authors' contributions}

W-TY, J-HW and C-FW conceived and designed research. X-LC and WZ conducted experiments. X-LC analyzed data. X-LC and WZ wrote the manuscript. C-WS, K-DY, T-MN, G-LY, XC, Y-LJ, J-ZW, H-BH, YZ, NW contributed to the work. All authors read and approved the final manuscript.

\section{Funding}

This work was supported by the National Key Research and Development Program of China (2017YFD0501000, 2017YFD0501200, 2017YFD0500400), the National Natural Science Foundation of China (32072888, 31972696 ,

$31941018,32072897)$ and Science and Technology Development Program of Jilin Province (20180201040NY, 20190301042NY).

\section{Availability of data and materials}

The raw data reported in the present paper have been deposited in Jilin Agricultural University, Chang Chun, China.

\section{Declarations}

\section{Ethics approval and consent to participate}

The whole animal experiment met the requirements of the Animal Management and Ethics Committee of Jilin Agricultural University.

\section{Consent for publication}

Not applicable.

Competing interests

The authors declare that there are no competing interests.

Received: 9 March 2021 Accepted: 5 Auqust 2021

Published online: 12 August 2021 


\section{References}

Aljurayyan A, Puksuriwong S, Ahmed M, Sharma R, Krishnan M, Sood S, Davies K, Rajashekar D, Leong S, McNamara P, Gordon S, Zhang Q (2016) Activation and induction of antigen-specific T follicular helper cells play a critical role in live-attenuated influenza vaccine-induced human mucosal anti-influenza antibody response. J Virol 92:e00114-e118

Argilaguet J, Pérez-Martín E, Nofrarías M, Gallardo C, Accensi F, Lacasta A Mora M, Ballester M, Galindo-Cardiel I, López-Soria S, Escribano J, Reche P. Rodríguez F (2012) DNA vaccination partially protects against African swine fever virus lethal challenge in the absence of antibodies. PLOS ONE 7:e40942

Arias M, Torre A, Dixon L, Gallardo C, Jori F, Laddomada A, Martins C, Parkhouse R, Revilla Y, Rodriguez F (2017) Approaches and perspectives for development of African swine fever virus vaccines. Vaccines 5:35

Attridge K, Kenefeck R, Wardzinski L, Qureshi O, Wang C, Manzotti C, Okkenhaug K, Walker L (2014) IL-21 promotes CD4 T cell responses by phosphatidylinositol 3-kinase-dependent upregulation of CD86 on B cells. J Immunol 192:2195-2201

Battaglia A, Buzzonetti A, Baranello C, Fanelli M, Fossati M, Catzola V, Scambia G, Fattorossi A (2014) Interleukin-21 (IL-21) synergizes with IL-2 to enhance T-cell receptor-induced human T-cell proliferation and counteracts IL-2/transforming growth factor- $\beta$-induced regulatory T-cell development. Immunology 139:109-120

Bonnet S, Bouhsira E, De RN, Fite J, Etoré F, Garigliany M, Jori F, Lempereur L, Potier ML, Quillery E, Saegerman C, Vergne T, Vial L (2020) Putative role of arthropod vectors in African swine fever virus transmission in relation to their bio-ecological properties. Viruses 12:778

Boyaka P (2017) Inducing mucosal IgA: a challenge for vaccine adjuvants and delivery systems. J Immunol 199:9-16

Cai R, Jiang Y, Yang W, Yang W, Shi S, Shi C, Hu J, Gu W, Ye L, Zhou F, Gong Q, Han W, Yang G, Wang C (2017) Surface-displayed IL-10 by recombinant Lactobacillus plantarum reduces Th1 responses of RAW264.7 cells stimulated with Poly(l:C) or LPS. J Microbiol Biotechnol 26:421-431

Calzas C, Chevalier C (2019) Innovative mucosal vaccine formulations against influenza A virus infections. Front Immunol 10:1605

Chege G, Burgers W, Müller T, Gray C, Shephard E, Barnett S, Ferrari G, Montefiori D, Williamson C, Williamson A (2017) DNA-MVA-protein vaccination of rhesus macaques induces HIV-specific immunity in mucosal-associated lymph nodes and functional antibodies. Vaccine 35:929-937

Chen X, Yang J, Ji Y, Okoth E, Liu B, Li X, Yin H, Zhu Q (2016) Recombinant Newcastle disease virus expressing African swine fever virus protein 72 is safe and immunogenic in mice. Virologica Sinica 31:150-159

Chen C, Hua D, Shi J, Tan Z, Zhu M, Tan K, Zhang L, Huang J (2020) Porcine immunoglobulin Fc fused P30/P54 protein of African swine fever virus displaying on surface of S. cerevisiae elicit strong antibody production in Swine. Virol Sin 36:207-219

Childerstone A, Takamatsu H, Yang H, Denyer M, Parkhouse R (1998) Modulation of T cell and monocyte function in the spleen following infection of pigs with African swine fever virus. Vet Immunol Immunopathol 62:281-296

Erman B, Bilic I, Hirschmugl T, Salzer E, Çagdas D, Esenboga S, Akcoren Z, Sanal O, Tezcan I, Boztug K (2015) Combined immunodeficiency with CD4 lymphopenia and sclerosing cholangitis caused by a novel loss-of-function mutation affecting IL21R. Haematologica 100:e216-219

Fan W, Jiao P, Zhang H, Chen T, Zhou X, Qi Y, Sun L, Shang Y, Zhu H, Hu R, Liu W, Li J (2020) Inhibition of African swine fever virus replication by porcine type I and type II interferons. Front Microbiol 11:1203

Fujimoto K, Kawaguchi Y, Shimohigoshi M, Gotoh Y, Nakano Y, Usui Y, Hayashi T, Kimura Y, Uematsu M, Yamamoto T, Akeda Y, Rhee J, Yuki Y, Ishii K, Crowe S, Ernst P, Kiyono H, Uematsu S (2019) Antigen-specific mucosal immunity regulates development of intestinal bacteria-mediated diseases. Gastroenterology 157:1530-1543

Gaudreault N, Richt J (2019) Subunit vaccine approaches for African swine fever virus. Vaccines 7:56

Hou Q, Ye L, Liu H, Huang L, Yang Q, Turner J, Yu Q (2018) Lactobacillus accelerates ISCs regeneration to protect the integrity of intestinal mucosa through activation of STAT3 signaling pathway induced by LPLs secretion of IL-22. Cell Death Differ 25:1657-1670

Huang $K$, Yang G, Jin Y, Liu J, Chen H, Wang P, Jiang Y, Shi C, Huang H, Wang J, Wang G, Kang Y, Yang W, Wang C (2018) Construction and immunogenicity analysis of Lactobacillus plantarum expressing a porcine epidemic diarrhea virus S gene fused to a DC-targeting peptide. Virus Res 247:84-93

Jia N, Ou Y, Pejsak Z, Zhang Y, Zhang J (2017) Roles of African swine fever virus structural proteins in viral infection. J Vet Res 61:135-143

Jiang Y, Yang G, Wang Q, Wang Z, Yang W, Gu W, Shi C, Wang J, Huang H, Wang C (2017) Molecular mechanisms underlying protection against H9N2 influenza virus challenge in mice by recombinant Lactobacillus plantarum with surface displayed HA2-LTB. J Biotechnol 259:6-14

Jin Y, Yang W, Shi C, Feng B, Huang K, Zhao G, Li Q, Xie J, Huang H, Jiang Y, Wang J, Wang G, Kang Y, Yang G, Wang C (2018) Immune responses induced by recombinant Lactobacillus plantarum expressing the spike protein derived from transmissible gastroenteritis virus in piglets. Appl Microbiol Biotechnol 102:8403-8417

Jin Z, Gao S, Cui X, Sun D, Zhao K (2019) Adjuvants and delivery systems based on polymeric nanoparticles for mucosal vaccines. Int J Pharm 572:118731

Lei H, Peng X, Zhao D, Ouyang J, Jiao H, Shu H, Ge X (2015) Lactococcus lactis displayed neuraminidase confers cross protective immunity against influenza A viruses in mice. Virology 476:189-195

Liu S, Luo Y, Wang Y, Li S, Zhao Z, Bi Y, Sun J, Peng R, Song H, Zhu D, Sun Y, Li S, Zhang L, Wang W, Sun Y, Qi J, Yan J, Shi Y, Zhang X, Wang P, Qiu H, Gao $\mathrm{G}$ (2019) Cryo-EM structure of the African swine fever virus. Cell Host Microbe 26:836-843.e3

Lokhandwala S, Petrovan V, Popescu L, Sangewar N, Elijah C, Stoian A, Olcha M, Ennen L, Bray J, Bishop R, Waghela S, Sheahan M, Rowland R, Mwangi W (2019) Adenovirus-vectored African swine fever virus antigen cocktails are immunogenic but not protective against intranasal challenge with Georgia 2007/1 isolate. Vet Microbiol 235:10-20

Lycke N (2012) Recent progress in mucosal vaccine development: potential and limitations. Nat Rev Immunol 12:592-605

Moens L, Tangye S (2014) Cytokine-mediated regulation of plasma cell generation: IL-21 takes center stage. Front Immunol 5:65

Muangkram Y, Sukmak M, Wajjwalku W (2015) Phylogeographic analysis of African swine fever virus based on the p72 gene sequence. Genet Mol Res 14:4566-4574

Ortega P, Saulle I, Mercurio V, Ibba S, Lori E, Fenizia C, Masetti M, Trabattoni D, Caputo S, Vichi F, Mazzotta F, Clerici M, Biasin M (2018) Interleukin 21 (IL-21)/microRNA-29 (miR-29) axis is associated with natural resistance to HIV-1 infection. AIDS 32:2453-2461

Oura C, Denyer M, Takamatsu H, Parkhouse R (2005) In vivo depletion of CD8+ T lymphocytes abrogates protective immunity to African swine fever virus. J Gen Virol 86:2445-2450

Petitdemange C, Kasturi S, Kozlowski P, Nabi R, Quarnstrom C, Reddy P, Derdeyn C, Spicer L, Patel P, Legere T, Kovalenkov Y, Labranche C, Villinger F, Tomai M, Vasilakos J, Haynes B, Kang C, Gibbs J, Yewdell J, Barouch D, Wrammert J, Montefiori D, Hunter E, Amara R, Masopust D, Pulendran B (2019) Vaccine induction of antibodies and tissue-resident CD8+T cells enhances protection against mucosal SHIV-infection in young macaques. JCI Insight 4:e126047

Petrovan V, Murgia M, Wu P, Lowe A, Jia W, Rowland R (2020) Epitope mapping of African swine fever virus (ASFV) structural protein, p54. Virus Res 279:197871

Rasheed M, Latner D, Aubert R, Gourley T, Spolski R, Davis C, Langley W, Ha S, Ye L, Sarkar S, Kalia V, Konieczny B, Leonard W, Ahmed R (2013) Interleukin-21 is a critical cytokine for the generation of virus-specific long-lived plasma cells. J Virol 87:7737-7746

Rock D (2017) Challenges for African swine fever vaccine development-". . perhaps the end of the beginning.". Vet Microbiol 206:52-58

Sánchez-Cordón P, Jabbar T, Berrezaie M, Chapman D, Reis A, Sastre P, Rueda P, Goatley L, Dixon L (2018) Evaluation of protection induced by immunisation of domestic pigs with deletion mutant African swine fever virus Benin $\triangle M G F$ by different doses and routes. Vaccine 36:707-715

Sang H, Miller G, Lokhandwala S, Sangewar N, Waghela S, Bishop R, Mwangi W (2020) Progress toward development of effective and safe African swine fever virus vaccines. Front Vet Sci 7:84

Shi S, Yang W, Yang G, Cong Y, Huang H, Wang Q, Cai R, Ye L, Hu J, Zhou J, Wang C, Li Y (2014) Immunoprotection against influenza virus H9N2 by the oral administration of recombinant Lactobacillus plantarum NC8 expressing hemagglutinin in BALB/c mice. Virology 464-465:166-176

Shoraka S, Mohebbi S, Hosseini S, Hosseini RA, Hatami Y, Sharifian A, Rostami-Nejad M, Asadzadeh AH, Zali M (2019) Association between Interleukin-21 and Interleukin-21 receptor gene polymorphisms with 
susceptibility to chronic hepatitis B virus infection and HBV spontaneous clearance in Iranian population. Microb Pathog 128:263-267

Sitt T, Kenney M, Barrera J, Pandya M, Eckstrom K, Warner M, Pacheco J, LaRocco M, Palarea-Albaladejo J, Brake D, Rieder E, Arzt J, Barlow J, Golde W (2019) Duration of protection and humoral immunity induced by an adenovirus-vectored subunit vaccine for foot-and-mouth disease (FMD) in Holstein steers. Vaccine 37:6221-6231

Takamatsu H, Denyer M, Lacasta A, Stirling C, Argilaguet J, Netherton C, Oura C, Martins C, Rodríguez F (2013) Cellular immunity in ASFV responses. Virus Res 173:110-121

Tangye S, Pillay B, Randall K, Avery D, Phan T, Gray P, Ziegler J, Smart J, Peake J, Arkwright P, Hambleton S, Orange J, Goodnow C, Uzel G, Casanova J, Lugo RS, Freeman A, Su H, Ma C (2017) Dedicator of cytokinesis 8-deficient CD4 T cells are biased to a T2 effector fate at the expense of T1 and T17 cells. J Allergy Clin Immunol 139:933-949

Tangye S, Ma C (2020) Regulation of the germinal center and humoral immunity by interleukin-21. J Exp Med 217:e20191638

Vinusha K, Deepika K, Johnson T, Agrawal G, Rakwal R (2018) Proteomic studies on lactic acid bacteria: a review. Biochem Biophys Rep 14:140-148

Wang L, Cui Y, Ruge F, Jiang WJ (2015) Interleukin 21 and its receptor play a role in proliferation, migration and invasion of breast cancer cells. Cancer Genomics Proteomics 12:211-221

Wang T, Sun Y, Qiu HJ (2018a) African swine fever: an unprecedented disaster and challenge to China. Infect Dis Poverty 7:111

Wang X, Wu J, Wu Y, Chen H, Zhang S, Li J, Xin T, Jia H, Hou S, Jiang Y, Zhu H, Guo X (2018b) Inhibition of CGAS-STING-TBK1 signaling pathway by DP96R of ASFV China 2018/1. Biochem Biophys Res Commun 506:437-443

Yang W, Yang G, Yang X, Shonyela S, Zhao L, Jiang Y, Huang H, Shi C, Wang J, Wang G, Zhao J, Wang C (2017) Recombinant Lactobacillus plantarum expressing HA2 antigen elicits protective immunity against H9N2 avian influenza virus in chickens. Appl Microbiol Biotechnol 101:8475-8484

Yang W, Li Q, Ata E, Jiang Y, Huang H, Shi C, Wang J, Wang G, Kang Y, Liu J, Yang G, Wang C (2018a) Immune response characterization of mice immunized with Lactobacillus plantarum expressing spike antigen of transmissible gastroenteritis virus. Appl Microbiol Biotechnol 102:8307-8318

Yang W, Yang G, Shi S, Liu Y, Huang H, Jiang Y, Wang J, Shi C, Jing Y, Wang C (2018b) Protection of chickens against H9N2 avian influenza virus challenge with recombinant Lactobacillus plantarum expressing conserved antigens. Appl Microbiol Biotechnol 101:4593-4603

Yang W, Yang G, Zhao L, Jin Y, Jiang Y, Huang H, Shi C, Wang J, Wang G, Kang Y, Wang C (2018c) Lactobacillus plantarum displaying conserved M2e and HA2 fusion antigens induces protection against influenza virus challenge. Appl Microbiol Biotechnol 102:5077-5088

Zhang Y, Zhou M, Wang Z, Yang J, Li M, Wang K, Cui M, Chen H, Fu Z, Zhao L (2016) Recombinant rabies virus expressing IL-21 enhances immunogenicity through activation of $\mathrm{T}$ follicular helper cells and germinal centre B cells. J Gen Virol 97:3154-3160

Zhao D, Liu R, Zhang X, Li F, Wang J, Zhang J, Liu X, Wang L, Zhang J, Wu X, Guan Y, Chen W, Wang X, He X, Bu Z (2019) Replication and virulence in pigs of the first African swine fever virus isolated in China. Emerg Microbes Infect 8:438-447

\section{Publisher's Note}

Springer Nature remains neutral with regard to jurisdictional claims in published maps and institutional affiliations.

\section{Submit your manuscript to a SpringerOpen ${ }^{\odot}$ journal and benefit from:}

- Convenient online submission

- Rigorous peer review

- Open access: articles freely available online

- High visibility within the field

- Retaining the copyright to your article

Submit your next manuscript at springeropen.com 